\title{
Study on the influence of indoor air particulate matter concentration in university classroom
}

\author{
Qi Cao \\ North China Electric Power University, Baoding 071000, China
}

15176275778@163.com

Keywords: indoor particulate matter concentration, PM2.5, PM10.0.

\begin{abstract}
This study mainly focuses on the concentration of particulate matter in different conditions. The classroom is selected as the object. By changing different conditions, the mass concentration and the number concentration of the PM10.0 and PM2.5 in the classroom were measured. Origin and SPSS are used to analyze the data to draw conclusions. Thus it is helpful for people to improve the concentration of particles in the classroom, which is of great significance to human health.
\end{abstract}

\section{Introduction}

Recently, indoor air quality has been paid more and more attention. More and more epidemiological studies have indicated that, even though the atmospheric particles concentration level is lower, there is still a significant correlation between the incidence rate and mortality of the population increase with the range of the relevant standards of the state [1-4]. On the other hand, in modern society, people spend almost $80 \%$ of the time in the room [5]. We can infer that the interior of the particle migration outside have a significant impact on human health. A large number of studies on the relationship between indoor and outdoor particulate matter pollutants show that the concentration of atmospheric particulate matter in the indoor environment is in the same order of magnitude as the concentrations of outdoor particulate matter. So it can be considered that the indoor environment is not only the most important, but also a very important atmospheric particulate matter exposure place. In this paper, the level of pollutants in the classroom under different conditions is studied. This paper has great practical significance, and innovative significance.

\section{Experiments and Methods}

Experiment instruments. The mass concentrations of PM2.5 and PM10.0 were measured by a smart dust detector (DUSTTRAK 8520). The number concentrations of the indoor particles were measured by a laser particle counter (8220 AEROTRAK) and its measurement range is greater than $0.3 \mu \mathrm{m}$. The experimental classroom is 8 meters long, 4 meters wide and 3 meters high. The instrument is 0.8 meters away from the ground.

Experimental scheme. The contents of the study were three: [1] the location factor; [2] the window factor; [3] the fan factor. The experiment time of the location factor was 220 minutes. The mass concentrations and the number concentrations of each position were measured 5 minutes before cleaning the blackboard. The four positions were the platform, the first row, the third row and the fifth row. These positions were $0.5 \mathrm{~m}, 1 \mathrm{~m}, 3 \mathrm{~m}$ and $5 \mathrm{~m}$. away from the blackboard. The mass concentrations and the number concentrations were measured 50min at each position when cleaning the blackboard one minute. The instruments are placed in the middle of each row.

The experiment time of the window factor was 100 minutes. The mass concentrations and the number concentrations were measured $10 \mathrm{~min}$ at each position, respectively, $0.2 \mathrm{~m}, 3 \mathrm{~m}$ and $6 \mathrm{~m}$ away from the window when window closed. After opening the window 40min, the mass concentrations and the number concentrations were measured $10 \mathrm{~min}$ at each position. The line of the three measurement sites was the horizontal line.

The experiment time of the fan factor was 30 minutes. The mass concentrations and the number concentrations were measured 5 minutes in the center of the classroom with the fan closed. After 
opening the fan 10min, the mass concentrations and the number concentrations were measured 10min at the same position.

\section{Results and Discussion}

\section{The effects of cleaning the blackboard}
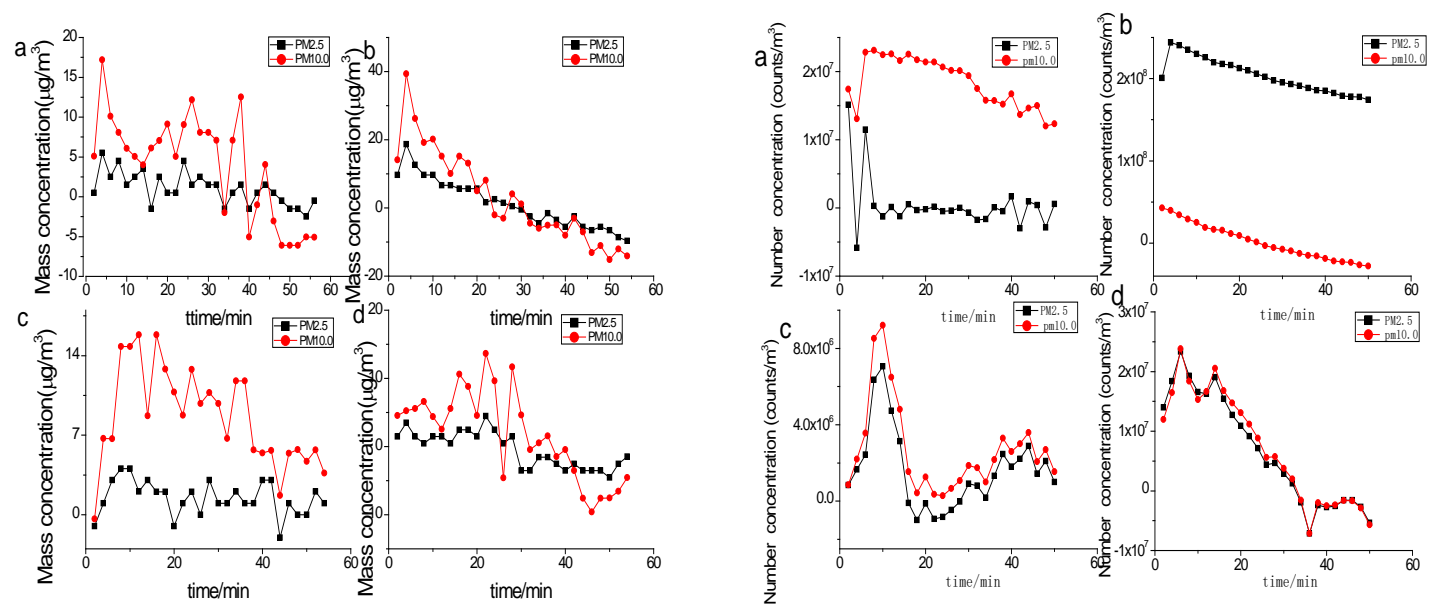

a-the platfom b-the first row c-the third row d-the fifth row

a-the platfom b-the first row c-the third row d-the fifth row

Fig. 1 The changes of mass concentration at different positions away from the blackboard

Fig.2 The changes of number concentration at different positions away from the blackboard

Fig. 1 shows the mass concentrations of the particles varied with time at four positions. Fig. 2 shows the number concentrations of the particles varied with time at four positions. Data in the figures is an increase in the amount of particulate matter after cleaning the blackboard. It can be seen from the pictures, in general, the particle concentration increases at each position and the closer the distance away from the blackboard, the average particle concentration higher. The podium and the first row reached the peak within 5 minutes, while the third row and fifth row reaches the peak more than 10 minutes. The closer the distance away from the blackboard, the shorter the time required to reach the peak value. It can also be seen that the change of PM10.0 is even greater than PM2.5. The level of the PM2.5 and PM10.0 0.5m away from the blackboard is the most unstable.

\section{The effects of opening the window}

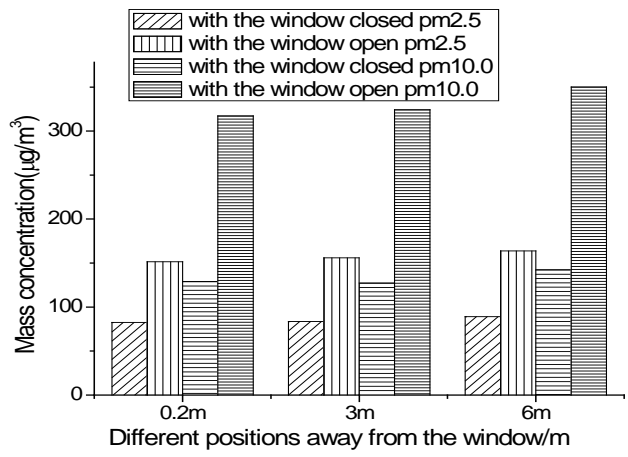

Fig.3 The changes of mass concentration at different positions away from the window

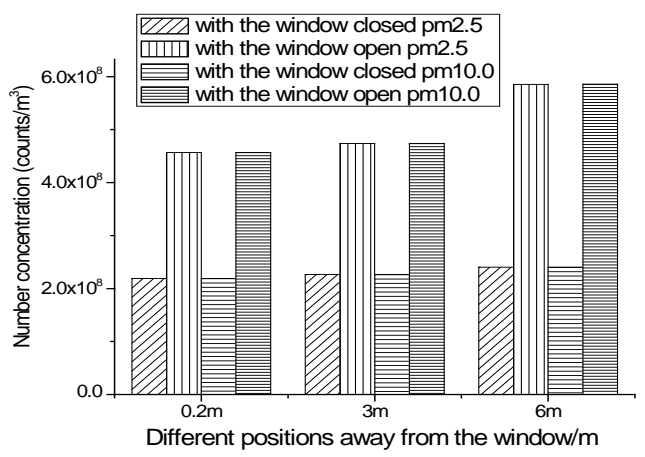

Fig.4 The changes of number concentration at different positions away from the window

Fig. 3 shows the mass concentrations of the particles at three positions. Fig. 4 shows the number concentrations of the particles at three positions. By the graph, it can be seen that the concentrations of particulate matter at different positions can increase after opening the window. The level of PM2.5 
increases almost two times. PM10.0 increases more than two times. It can be seen that the level of PM2.5 and PM10.0 of the position 6m away from is highest.

Table.1 Correlation analysis between PM2.5, PM10.0 and distance from the window

\begin{tabular}{cccc}
\hline & & Distance & PM2.5 \\
\hline Distance & Pearson relativity & 1 & $0.804^{* *}$ \\
& Significance(two sides) & & 0.000 \\
PM2.5 & N & 15 & 15 \\
& Pearson relativity & $0.804^{* *}$ & 1 \\
& Significance(two sides) & 0.000 & 15 \\
PM10.0 & N & 15 & 1 \\
& Person relativity & 0.817 & \\
& Significance(two sides) & 0.000 & 15 \\
\hline
\end{tabular}

Table 1 shows that the correlation analysis between PM2.5, PM10.0 and distance away from the window. It can be seen that the correlation coefficient between the PM2.5 and the distance away from the window is 0.804 , which is higher than 0.8 . That is to say, it is highly relevant. It can also be seen that the correlation coefficient between the PM10.0 and the distance away from the window is 0.817 . It can draw conclusions that the level of PM2.5 and PM10.0 is highly correlation with the distance away from the window.

The effects of opening the fan

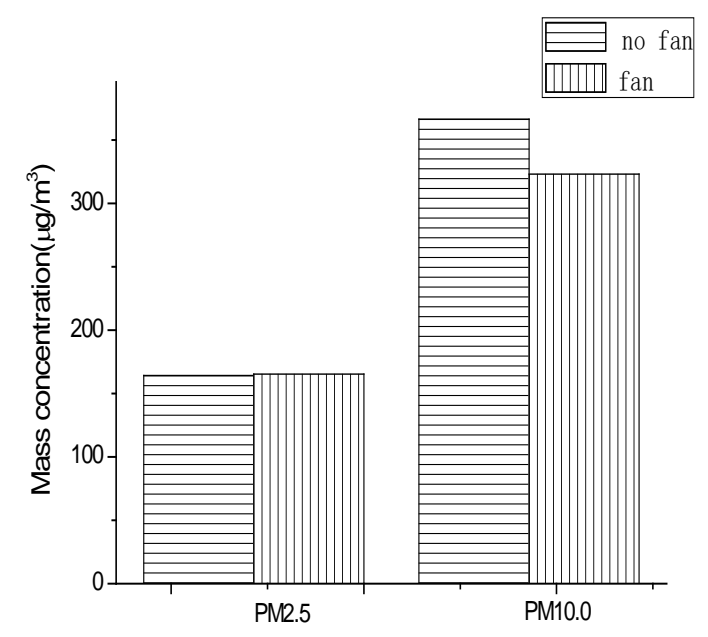

Fig. 5 The changes of mass concentration after opening the fan

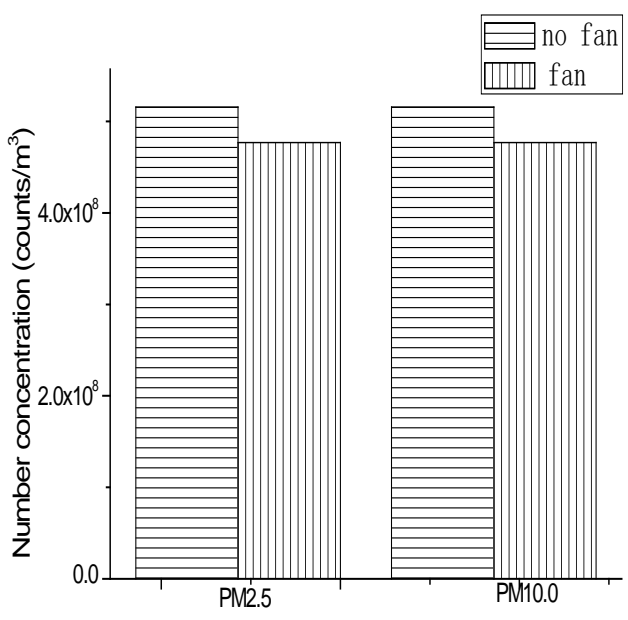

Fig.6 The changes of number concentration after opening the fan

From the Fig.5, it can be seen that PM2.5 has little change in mass concentration. The mass concentration of PM10.0 decreases clearly. It can also be seen from the Fig. 6 that the number concentration of PM2.5 and PM10.0 both decrease.

\section{Summary}

This paper studied the influence of indoor air particulate matter concentration in the university classroom. The conclusions are shown as follows.

(1) Cleaning the blackboard will increase level of PM2.5 and PM10.0 in the classroom.

(2) After cleaning the blackboard, the location closer to the blackboard, the average lever of PM2.5 and PM10.0 is higher. 
(3) The closer to the blackboard, the shorter the time required to reach the peak of PM10.0 and PM2.5.

(4) After opening the window, the farther the distance away from the window, the higher the level of PM10.0 and PM2.5 is.

(5) The levels of PM2.5 and PM10.0 are correlated to the distance away from the window highly, and the correlation coefficients are more than 0.8 .

(6) Opening the fan can decrease the mass concentration and the number concentration of PM2.5 and PM10.0 to a certain extent.

According to the conclusions, some methods can be taken to improve the environment in the classroom. When the PM2.5 outdoor is high, it is better to close the window. If it is needed to open the window, the position near the window is the best choice. In summer, it is better to open the fan, which can increase the air change but also reduce the concentration of particles in class.

\section{Acknowledgments}

This research has been supported by "College students innovation and entrepreneurship training program” (Grant No.20142086).

\section{Reference}

[1] C. A. Pope, D. V. Bates, M. E. Raizenne, Health effects of particulate air pollution, time for reassessment, J. Environmental Health Perspectives. 1995 (103) 472-480.

[2] D.W. Dockery, Health effects of particulate air pollution, J. Annals of Epidemiology. 19(2009) 257-263.

[3] H. Kan, S.J. London, G. Chen, Y. Zhang, G. Song, N. Zhao, L. Jiang, B. Chen, Season, sex, age, and education as modifiers of the effects of outdoor air pollution on daily mortality in Shanghai, China: The Public Health and Air Pollution in Asia (PAPA) Study, J. Environmental Health Perspectives. 116 (2008) 1183-1188.

[4] S.A. Venners, B. Wang, Z. Xu, Y. Schlatter, L. Wang, X. Xu, Particulate matter, sulfur dioxide, and daily mortality in Chongqing, China, J. Environmental Health Perspectives. 111(2003) 562-567.

[5] C. Monn, Exposure assessment of air pollutants: A review on spatial heterogeneity and indoor/outdoor/personal exposure to suspended particulate matter, nitrogen dioxide and ozone, J. Atmospheric Environment. 35 (2001) 1-32. 